\title{
Germanica
}

les arts dans les pays germanophones au $\mathrm{XX}^{\mathrm{e}}$ siècle

\section{Positions artistiques en RDA : le Sisyphe de Mattheuer}

Artistic positions in the GDR: Mattheuer's Sisyphus

Künstlerische Stellungen in der DDR: Wolfgang Mattheuers Sisyphios

\section{Stefanie Schwabe}

\section{OpenEdition \\ Journals}

Édition électronique

URL : http://journals.openedition.org/germanica/826

DOI : $10.4000 /$ germanica.826

ISSN : 2107-0784

\section{Éditeur}

Université de Lille

\section{Édition imprimée}

Date de publication : 30 décembre 2009

Pagination : 73-86

ISBN : 978-2-913857-24-7

ISSN : 0984-2632

Référence électronique

Stefanie Schwabe, «Positions artistiques en RDA : le Sisyphe de Mattheuer », Germanica [En ligne], 45 2009, mis en ligne le 01 décembre 2011, consulté le 06 octobre 2020. URL : http://

journals.openedition.org/germanica/826 ; DOI : https://doi.org/10.4000/germanica.826

Ce document a été généré automatiquement le 6 octobre 2020.

(C) Tous droits réservés 


\title{
Positions artistiques en RDA : le Sisyphe de Mattheuer
}

\author{
Artistic positions in the GDR: Mattheuer's Sisyphus \\ Künstlerische Stellungen in der DDR: Wolfgang Mattheuers Sisyphios
}

\section{Stefanie Schwabe}

1 Cet article se concentre sur trois tableaux ${ }^{1}$ de Wolfgang Mattheuer qui compte parmi les artistes emblématiques du paysage artistique de la RDA. Son nom est irrévocablement lié à l'École de Leipzig ${ }^{2}$, qui aux yeux du public étranger incarna la RDA et sa doctrine du réalisme socialiste. Pourtant, Mattheuer faisait également partie des artistes dont les œuvres déclenchèrent des débats publics de nature à exercer une influence réelle sur le discours officiel sur l'art.

2 Grâce à la popularité et à la reconnaissance officielle dont il jouissait, Mattheuer a pu bénéficier de privilèges, et notamment d'une relative liberté d'expression dans ses discours et dans son œuvre picturale. Par leur simplicité technique, leur style réaliste et leurs contenus sociaux, ses œuvres témoignent d'un travail artistique critique et profondément subversif.

3 À partir de 1965, Mattheuer commence par reprendre l'épisode biblique de Caïn, mais aussi des allégories, puis des récits de la mythologie antique. Par le recours aux mythes universaux de l'humanité (Icare, Sisyphe et Prométhée), Mattheuer (ré)introduit les problèmes collectifs et individuels de l'homme, et l'expression de son malaise dans la société moderne.

4 Dès la fin des années 1960, sont engagées en RDA les premières réflexions théoriques sur la réception de l'héritage culturel de l'Antiquité dans les arts, notamment dans la littérature, le théâtre et les arts plastiques. Cette réflexion est inaugurée par le congrès sur Das klassische Altertum in der sozialistischen Kultur [L'Antiquité classique dans la culture socialiste] en janvier $1969^{3}$, lors duquel les intervenants posent le problème de l'actualisation des sujets de l'Antiquité classique, en relation avec la culture socialiste.

5 L'introduction de la réception de l'Antiquité classique dans le discours officiel des arts en RDA est étroitement liée à l'histoire de la ville de Stendal (aujourd'hui dans le Land 
de Saxe-Anhalt) qui héberge le musée Winckelmann. Depuis 1971, celui-ci s'est efforcé de constituer une collection de documents en rapport avec la réception de l'Antiquité en RDA, dans les domaines des arts plastiques, du théâtre et de la littérature. Celle-ci compte aujourd'hui, environ 750 œuvres, en grande partie des dessins et des estampes, mais aussi des peintures et des sculptures ${ }^{4}$. Depuis 1974, ce musée, spécialisé dans la réception de l'art antique, publie des ouvrages scientifiques. Pour les arts en général, la deuxième moitié des années 70 est marquée par la multiplication des motifs empruntés aux mythologies antiques. En ce qui concerne les arts plastiques, les figures les plus remarquables sont Hercule ${ }^{5}$, Icare et Prométhée $e^{6}$, plus rares sont des sujets tels que la chute de Phaéton ou les travaux de Sisyphe ${ }^{7}$.

6 L'introduction de ces figures de la mythologie antique permettait aux artistes de traduire sur le plan pictural le sujet de l'homme moderne en quête du Moi. L'homme cherche, dans un monde dépourvu de moyens de communiquer qui pourrait l'aider à affronter l'inconscient collectif, à accomplir un processus d'individuation. Selon Carl Gustav Jung, il s'agit d'un processus psychologique qui fait de l'être humain un individu. Le contenu de l'idéologie marxiste-léniniste inhérente à la société socialiste de la RDA s'oppose à une telle démarche de réflexion intérieure. Mattheuer s'est servi ainsi des mythes antiques pour dénoncer le dysfonctionnement de la société estallemande et la position des artistes et des individus au sein de cette société.

Dans ses œuvres consacrées au mythologème de Sisyphe aux Enfers, Mattheuer s'inspire non seulement des récits antiques, mais aussi de la réalité sociale en RDA. De plus, l'essai Le Mythe de Sisyphe d'Albert Camus de 1942 semble avoir eu une forte influence sur la production artistique de Mattheuer pour les trois oeuvres traitées dans cet article. La référence picturale à ce texte témoigne de la réception de la pensée politique non-conformiste de Camus en RDA. La critique d'art est-allemande des années 70 évoque la réception de Camus par Mattheuer, sans en approfondir le sujet. Selon l'historien d'art Dieter Gleisberg, le Sisyphe de Mattheuer se distinguerait de celui de Camus, par le refus du personnage de se soumettre à son destin. Pourtant, le Sisyphe de Mattheuer rejoint par de nombreux détails celui du texte littéraire de Camus, car il ne semble pas illustrer uniquement l'homme qui s'affranchit d'un travail répétitif et inutile, même si la critique d'art est-allemande de l'époque veut nous le faire croire. Cette confusion sur le contenu résulte probablement de l'impossibilité d'accéder à la traduction ouest-allemande du Sisyphe camusien en RDA.

8 L'historien d'art Ulrich Kuhirt ${ }^{8}$ propose en 1975 une interprétation beaucoup plus restrictive mais conforme au système politique de RDA: Sisyphe se libérerait d'un travail improductif afin d'atteindre le bonheur par un travail utile. Selon Kuhirt toute autre interprétation des œuvres de Mattheuer serait aberrante. En 1979, Mattheuer reprend dans son discours artistique cette première interprétation selon laquelle Sisyphe symboliserait avant tout une forme de travail inutile?

9 Pourtant, le Sisyphe de Mattheuer est aussi moderne et complexe que celui de Camus. Ce dernier attribue au héros antique la faculté de penser et de se révolter. Le Sisyphe de Mattheuer se caractérise également par sa volonté de déclencher une révolte absurde et d'obtenir une liberté éphémère.

10 Au début des années 80 , l'historien d'art Gerd H. Zuchold ${ }^{10}$ propose dans la préface du catalogue d'exposition Das Erbe der Antike [L'Héritage de l'Antique ${ }^{11}$ une interprétation plus osée du Sisyphe de Mattheuer. Ce dernier doit, selon Zuchold, subir les effets du pouvoir tant que celui-ci ne transforme pas ses structures. Il l'interprète donc 
indirectement comme un Sisyphe profondément subversif. Le premier tableau de la série, Die Flucht des Sisyphos [La Fuite de Sisyphe], aurait dû être exposé en 1980 à BerlinEst mais fut retenu pour une exposition en Suède.

11 Les trois œuvres de Mattheuer, réalisées en 1972, 1974 et 1975, sont une réponse humaine et un reflet des stratégies politiques de la RDA. Il s'agit de la brève période d'ouverture culturelle au début de la décennie, sensible avant tout dans le discours officiel, marquée par une politique de détente ainsi que des échanges artistiques avec l'autre Allemagne et l'étranger « non-socialiste ». Au niveau artistique, on constate une intégration théorique des formes d'art avant-gardiste, comme le collage, dans le discours officiel sur le réalisme socialiste ${ }^{12}$.

La contrepartie de cet esprit d'ouverture est le raidissement dans la politique culturelle. C'est l'époque où le ministère de l'Intérieur développe l'appareil de surveillance de la Stasi. Cette hypocrisie politique, sous le masque d'une certaine liberté d'expression artistique, prend fin brutalement avec l'expatriation de l'auteurinterprète Wolf Biermann, déchu de la nationalité est-allemande en novembre 1976. Cet événement marque un tournant dans la vie artistique des années 1970. En effet, depuis 1977, les citoyens de la RDA peuvent demander légalement leur expatriation ${ }^{13}$ et beaucoup d'artistes font usage de cette possibilité.

Les trois peintures de Mattheuer représentant le mythologème de Sisyphe aux Enfers nous interpellent par leur universalité et leur actualité.

Les écrits de l'Antiquité grecque (Iliade et Odyssée de Homère) et romaine (Métamorphoses d'Ovide), décrivent le personnage de Sisyphe de manière complexe mais aussi contradictoire. Il s'agit d'un personnage qui essaie de capturer la mort, poussé par sa passion de la vie et qui est puni par les dieux pour avoir osé les défier. Il est condamné à rouler un rocher ${ }^{14}$ jusqu'en haut d'une colline, dans le Tartare, lieu des Enfers. Mais sous l'effet de la puissance des dieux, le rocher dégringole chaque fois avant même d'atteindre le sommet. Sisyphe est ainsi sanctionné par un travail apparemment dépourvu de sens et absurde, puisque le but n'est jamais atteint. Sisyphe n'arrive pas à se libérer de ce travail forcé ordonné par des puissances supérieures et il est conscient de cet interminable châtiment. C'est sur ce dernier point que le Sisyphe moderne se distingue de celui de l'Antiquité ${ }^{15}$. Le Sisyphe de Mattheuer n'accepte pas son sort, il se révolte. Non seulement il est conscient de son existence absurde, mais il cherche également à dépasser cette crise. Les trois tableaux de Mattheuer illustrent trois manières de refuser la situation donnée : la fuite, la création et la dérision. L'activité répétitive de Sisyphe est interrompue par ces péripéties et donne de nouvelles directions à son existence. Mattheuer ne donne pas une interprétation artistique définitive du mythologème et nous laisse sans réponse quant à l'aboutissement du processus d'individuation de son Sisyphe.

15 À l'examen de ces trois œuvres, on observe que Mattheuer intègre toujours ses sujets dans un paysage contemporain du processus de leur création. Ces paysages évoquent principalement la région du Vogtland, patrie de Mattheuer, caractérisée par un relief plutôt montagneux et par la présence de sites industriels. Le Sisyphe moderne de Mattheuer n'est donc pas situé aux Enfers comme le Sisyphe imaginaire des mythologies antiques, mais dans la RDA des années 1970.

Le langage pictural de Mattheuer se traduit par un réalisme proche de ce qui a été officiellement défini comme réalisme socialiste. Son travail figuratif et narratif cerne l'homme comme sujet central. Mais, en général, quelques détails de caractère 
surréaliste attirent l'attention et permettent d'engager un dialogue narratif entre l'œuvre et le public. Précisément, pour deux des trois œuvres évoquées, il s'agit du masque de mouton que revêt d'abord un personnage isolé et anonyme ( $L a$ fuite de Sisyphe); on retrouve ensuite ce masque abandonné sur le sol sans qu'il soit possible pour autant d'identifier le porteur du masque, car il se fond dans un groupe de plusieurs individus (Sisyphe impétueux et les siens).

Dans le théâtre antique, le masque joue un rôle primordial. La persona ${ }^{16}$ servait à marquer le caractère du rôle et c'est par elle que passait la voix de l'acteur. Carl Gustav Jung reprend cette notion dans sa psychologie analytique pour désigner la partie de la personnalité qui organise les rapports de l'individu à la société afin de tenir un rôle social prédéfini. Ce masque social est l'interstice entre l'identité extérieure collective et l'identité intérieure individuelle.

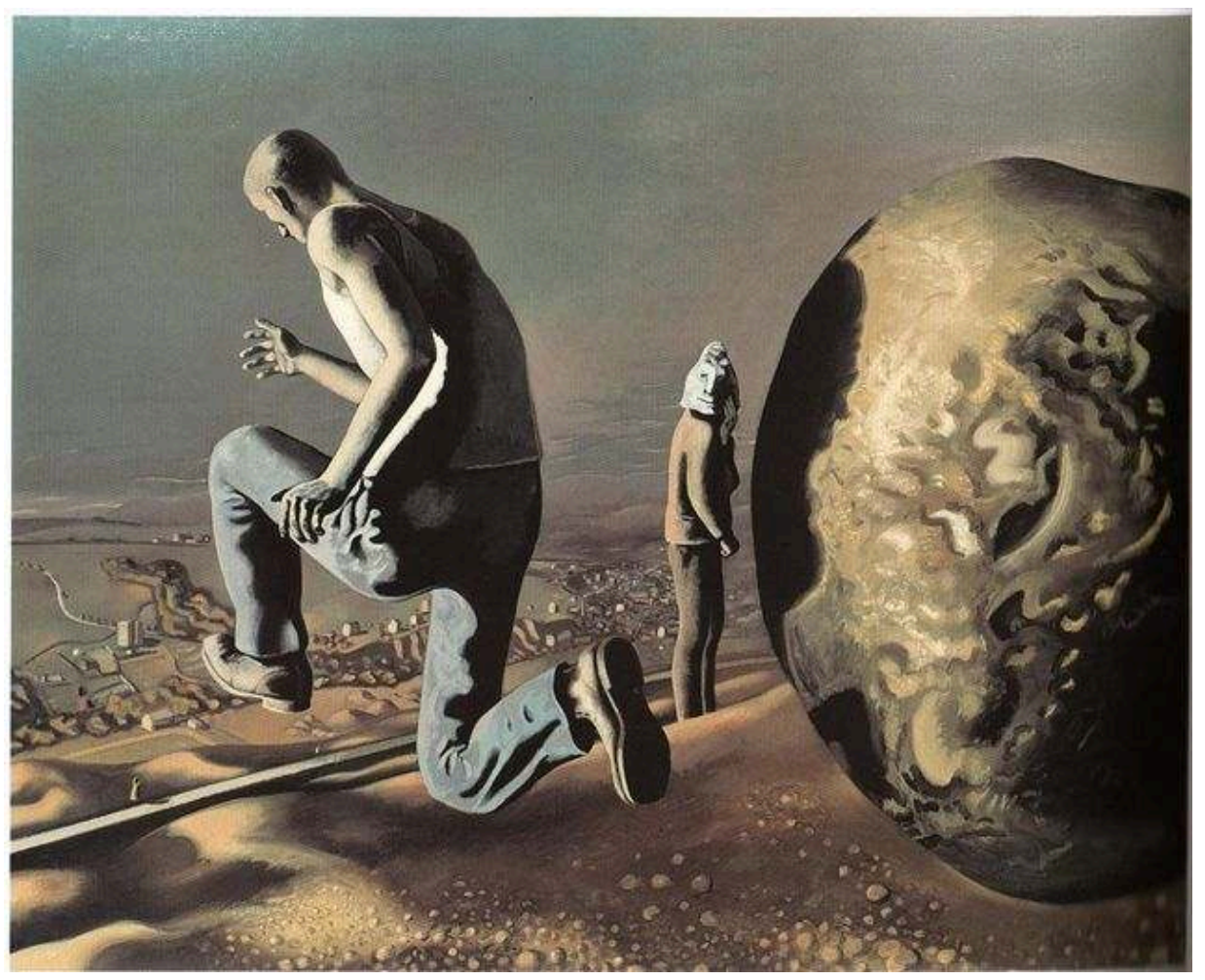

Le premier tableau intitulé La Fuite de Sisyphe date de 1972. C'est l'année où le peintre perce sur la scène artistique grâce à deux expositions d'art contemporain, celle de la circonscription de Leipzig et la $7^{e}$ exposition d'art de la RDA à Dresde. Mais cette première grande reconnaissance publique n'est pas due à l'œuvre citée qui, de fait, sera exposée pour la première fois en 1974 à Leipzig, puis à Dresde lors de la première grande exposition personnelle de l'artiste ${ }^{17}$.

Dans ce premier tableau consacré à Sisyphe, Mattheuer représente le condamné dans un paysage à l'horizon large et profond, qui se déploie dans une perspective à vol d'oiseau ; l'observateur/-trice se trouve au même niveau que le personnage principal, quasiment au sommet de la colline. Sisyphe, en tenue d'ouvrier, descend la colline en courant vers un village industriel afin d'échapper au grand rocher à taille humaine dévalant lui aussi la pente. 
Le deuxième tableau Sisyphe taille le rocher, datant de 1974, représente un Sisyphe en vêtements de bureaucrate taillant la forme d'un poing fermé dans le rocher, symbolisant la résistance aux contraintes imposées, c'est-à-dire à la politique estallemande de l'époque. Cette fois encore, le paysage est industriel avec, à l'arrière-plan, une usine chimique et au premier plan une décharge où Sisyphe travaille le rocher. L'observateur se trouve, à nouveau, au même niveau que le personnage principal, c'està-dire au pied de la décharge. Au second plan, trois personnages, derrière une barrière, regardent l'homme qui travaille.

La décharge symbolise la société de consommation. Sur le tableau on voit des tuyaux et une carcasse de voiture de couleur éclatante. Les déchets envahissent le tableau, et leur couleur sombre suggère la saleté, le dégoût, le désespoir. Devant cet arrière-plan morbide se trouve un Sisyphe bureaucrate occupé à tailler le rocher. C'est dans ce lieu d'accumulation de tous les déchets de la société moderne, qui suggère en quelque sorte l'inconscient collectif, que Sisyphe s'installe afin de transformer les structures du pouvoir par son acte créateur. Dans la scène, les trois observateurs restent à l'écart, séparés de la décharge et de l'artiste par une simple barrière franchissable à tout moment. Ils peuvent donc rejoindre Sisyphe, lucide et conscient de son acte, afin de participer à son travail ou de le détruire. L'individu anonyme et passif observant la 
scène du premier tableau a été remplacé ici par un groupe de trois individus également passifs, mais susceptibles d'entrer en action à tout moment.

Ce tableau de 1974 représente l'homme qui transforme les conditions inhérentes à son existence par sa créativité. Mattheuer représente ainsi l'une des positions artistiques les plus répandues en RDA, celle de l'artiste comme acteur/-trice responsable participant à la transformation de la société par le biais de son art et de son travail individuels.

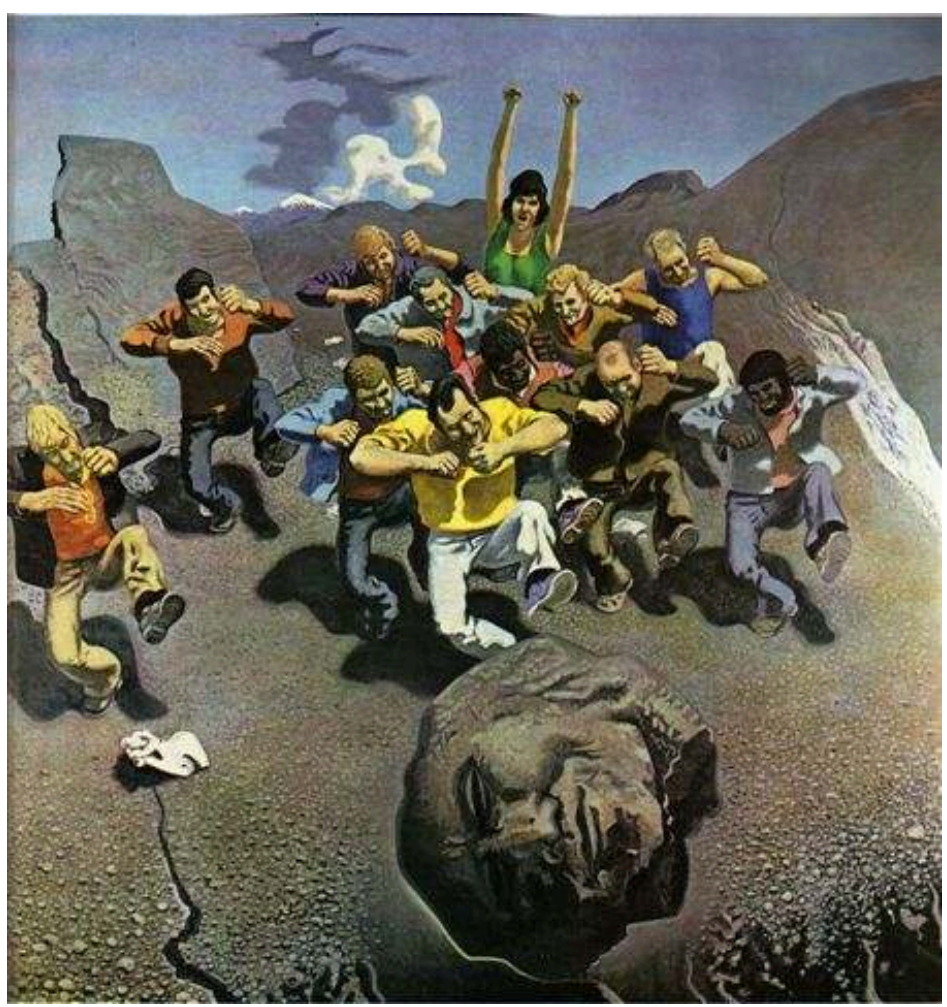

Le tableau Sisyphe impétueux et les siens de 1975 faisait partie des six peintures que Mattheuer a pu présenter en 1977 à la Documenta 6 de Kassel ${ }^{20}$. Sa participation a permis de faire connaître sa peinture à un large public. Dans la presse internationale, la participation officielle de six artistes est-allemands à la Documenta rencontre un écho assez défavorable, à cause du statut politique de la RDA. Pourtant, ce tableau de Mattheuer, chargé d'éléments fortement subversifs, faisait apparaître une critique très claire du système est-allemand. En RDA, l'œuvre fut reproduite en 1977 dans le magazine officiel de l'union des artistes plasticiens Bildende Kunst et exposée en 1988 à Berlin-Est, à l'occasion d'une grande exposition intitulée Nähe und Horizont [Proximité et Horizon].

Dans cette scène, Sisyphe, en tenue de loisirs, est accompagné d'un groupe de personnages dans un paysage toujours montagneux et industriel. Sisyphe semble très détendu et descend la colline en dansant et courant derrière le rocher taillé en forme de portrait. Tous les personnages qui l'accompagnent ont l'air insouciant. Ils sont en train de se moquer du pouvoir répressif représenté par la tête sculptée dont l'identité exacte est difficile à préciser. Selon Zuchold, les personnages ne seraient pas conscients des structures de pouvoir auxquelles Sisyphe est soumis, d'où leur incapacité à changer la situation absurde ${ }^{21}$. Mattheuer représente un Sisyphe engagé dans un processus de prise de conscience via la dérision qui fonctionne ici comme une forme de la distanciation critique volontaire. Le masque de mouton que le personnage isolé porte 
sur le premier tableau de la série a été ôté et gît à terre. L'individu ne se cache plus derrière un masque mais se fond dans la masse en imitant les mêmes gestes répétitifs du groupe triomphant et apparemment solidaire. Albert Camus écrit en 1951 dans L'Homme révolté «La solidarité des hommes se fonde sur le mouvement de révolte et celui-ci, à son tour, ne trouve de justification que dans cette complicité. » Sisyphe n'agit donc plus seul, mais il est encouragé par un groupe de personnes qui soutiennent ses actes de libération.

La scène est encadrée par un paysage montagneux et rocheux dépouillé de toute végétation. Le contenu de ce tableau de 1976 semble anticiper les événements de 1989. Sisyphe impétueux et les siens se révoltent pacifiquement et solidairement. Un groupe de 10 hommes de différents âges et origines et une femme en tenue claire de loisirs accompagnent Sisyphe. La femme se trouve derrière le groupe et tend vers le ciel ses deux bras aux poings fermés. La présence de cette femme encourageant par ses cris le groupe d'hommes renvoie à l'image du personnage féminin du tableau La Liberté guidant le peuple de Delacroix (1830).

Le Sisyphe moderne de Mattheuer est démystifié et profondément humain dans la représentation de sa recherche d'émancipation des structures de pouvoir inhérente à sa condition existentielle et à sa recherche du Moi. Le Sisyphe de Mattheuer fuit d'abord l'absurdité de la réalité, ce qui n'est pas une solution à long terme de ses problèmes. Puis, il se libère de son activité insensée et inutile, d'abord par l'acte individuel et créateur, puis ensuite, par l'acte collectif de la révolte. Les deux premiers tableaux ne donnent aucun indice sur les conséquences à long terme de ses actes de libération, notamment la fuite et l'appropriation du rocher. Que se passe-t-il après le moment de la libération ? À l'arrière-plan du troisième tableau, on peut apercevoir un personnage isolé en train de remonter un rocher sur la même colline. Ainsi la conquête de la liberté ne serait qu'éphémère et ne permettrait pas l'émancipation d'un travail absurde. Il faudrait toujours affronter le destin dans une lutte répétitive et éternelle.

Aucun des trois tableaux sur le thème de Sisyphe n'a été montré lors d'une des grandes expositions d'art de la $\mathrm{RDA}^{22}$. En 1981, Mattheuer était présent avec huit œuvres à l'exposition Peinture et gravure en RDA au Musée d'Art Moderne de la ville de Paris. Trois de ses peintures phares, Horizon (1970), Par-delà les sept montagnes (1973) et La travailleuse décorée (1974) y étaient exposées. Aucune œuvre faisant référence à la réactualisation des mythes antiques, comme les travaux de l'artiste sur Sisyphe, Icare ou encore Prométhée, ne figurait dans l'exposition. Par contre, on y trouvait une gravure sur bois intitulée Rencontre déconcertante ${ }^{23}$ (1976) où figure un groupe de quatre personnages ressemblant fortement par leurs mouvements au groupe solidaire de Sisyphe impétueux et les siens. Cette œuvre, également exposée en 1977 à Dresde lors de la $8^{e}$ exposition d'art de la RDA, donne une réplique angoissante au tableau, car ce groupe de personnages est attendu par deux personnes dont on aperçoit uniquement les ombres menaçantes, suggérant l'omniprésence des représentant(e)s du pouvoir. Mattheuer fait probablement allusion à la Stasi dont le rôle s'accrut durant l'ère Honecker (1971-1989). Pendant la période de la RDA et malgré son statut au sein du parti de la SED, l'artiste fut lui même victime de l'appareil de surveillance évoqué par cette gravure.

31 De nombreux artistes de la génération suivante, élèves ou non de Mattheuer, ont inauguré un langage symbolique personnel en référence à l'antique, à l'histoire allemande, à la situation politique dans la RDA des années 1970 et 1980. 
Dagmar Ranft-Schinke (1944), élève de Mattheuer, se consacre depuis 1968 au motif du Pégase issu de la mythologie greque. L'artiste représente dans son art, qu'elle caractérise elle-même de réalisme visionnaire, une créature survolant un monde visionnaire reflétant la réalité telle qu'elle est perçue par l'artiste. Ce motif est récurrent dans son travail artistique, qui s'inscrit dans une certaine continuité et qui exprime son amour profond pour la nature, notamment les chevaux ${ }^{24}$.

Une autre œuvre remarquable est celle du peintre et artiste Lutz Dammbeck qui travailla également, comme Dagmar Ranft-Schinke, sur le sujet des métamorphoses et qui initia au début des années 80 son concept Héraclès. Dammbeck étudia également à l'école d'art de Leipzig, dans la classe de Heinz Wagner. Ce concept artistique comprend un « média collage » intitulé La Caverne d'Héraclès (1983-1990) dans lequel l'artiste nous raconte l'existence du héros antique transposé dans la société moderne et toujours à la recherche du Moi par le biais d'une biographie fictive évoquant, entre autre, l'histoire douloureuse de l'Allemagne. L'artiste n'a pu finaliser ce "média collage » qu'après la chute du Mur.

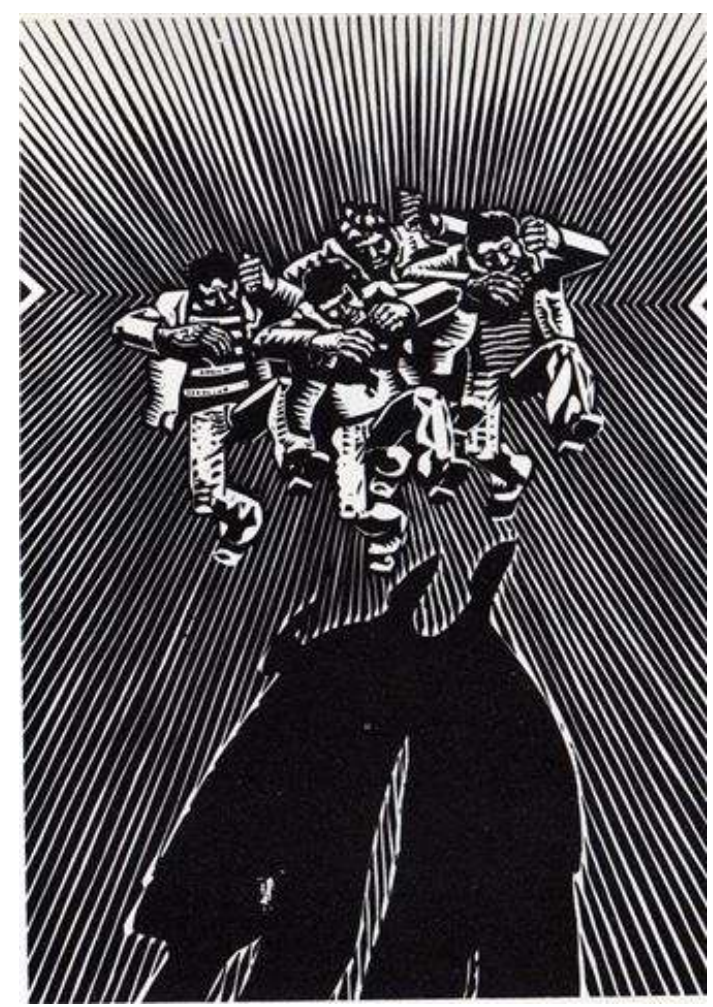

Mattheuer disparaît en 2004, le jour de son $77^{\mathrm{e}}$ anniversaire. Il laisse derrière lui une multitude d'œuvres caractérisées par leur symbolisme universel et personnel, inspiré par l'art antique, mais aussi par l'art romantique. Son langage pictural réaliste lui permettait de transmettre des valeurs humaines à travers une œuvre symbolique, parfois surréaliste. Sa position artistique permet de la définir comme critique et subversive et elle mérite pleinement d'être intégrée dans une histoire de l'art européenne élargie. 


\section{BIBLIOGRAPHIE}

«Sisyphos », in Lexikon der Kunst, vol. IV, Leipzig : Seemann, 1977, p. 519-520.

ARLT Peter, Antikerezeption in der bildenden Kunst der DDR : zu den Entwicklungsprozessen der antikmythologischen Ikonografie in Malerei, Grafik und Plastik von 1945-1985 und der ikonografischikonologischen Methode in den Kunstwissenschaften der DDR, Erfurt-Mühlhausen : Pädag. Hochsch., Diss. B, [1985], non paginé. [mémoire de thèse].

CAMUS Albert, Le mythe de Sisyphe - Nouvelle édition augmentée d'une étude sur Franz Kafka, [Paris] : Gallimard, 1945, 189 p., (Les Essais (Paris, 1931), ISSN 0768-4355 ; 12).

COLLARD Maggy, «Sisyphe : Histoire d'une liberté », in Folia Electronica Classica, n³, jan-juin 2002. [http://bcs.fltr.ucl.ac.be/fe/03/Sisyphe.html]

GLEISBERG Dieter, « Wolfgang Mattheuer », in Bildende Kunst, n6, 1977, p. 278.

IRMSCHER Johannes, Antikerezeption, deutsche Klassik und sozialistische Gegenwart, Berlin [Est] : Akademie-Verlag, 1979, 15 p., (Schriften der Winckelmann-Gesellschaft ; 5).

IRMSCHER Johannes, Das Antikebild unserer Gegenwart : Tendenzen und Perspektiven, Berlin [Est] : Akademie-Verlag, 1979, 23 p., (Sitzungsberichte der Akademie der Wissenschaften der DDR / G ; 1979,4). [Vortrag gehalten in der wissenschaftlichen Sitzung des Plenums der Akademie der Wissenschaften der DDR am 23. Februar 1978].

IRMSCHER Johannes, Probleme der Aneignung des antiken Erbes, Berlin [Est] : Akademie-Verlag, $1976,30 \mathrm{p}$.

JUNG Carl Gustav ; Charles KERENYI, Introduction à l'essence de la mythologie, Paris : Payot, 2001 [1941], $284 \mathrm{p}$.

JUNG Carl Gustav, « La Naissance du héros », in L'homme à la découverte de son âme : structure et fonctionnement de l'inconscient La Réalité de l'âme, Paris : Michel, 1987 [1928-34], p. 407-443.

KUHIRT Ullrich, « Ein ungewöhnlicher Realismus? », in Bildende Kunst, n6, 1975, p. 281-285.

KUNZE Max, Antikerezeption heute: Protokoll eines Kolloquiums, Stendal : Winckelmann-Gesellschaft, 1985, 118 p., (Beiträge der Winckelmann-Gesellschaft Stendal ; 13).

KUNZE Max, Catalogue Antikerezeption in der Kunst der DDR: Rückblicke, Stendal : WinckelmannGesellschaft, 1991, 32 p.

RITTIG Roland ; Rüdiger ZIEMANN, Prometheus 1982 : unbeliebte Kunst aus der DDR, Halle ; Zurich : Janos Stekovics, 1995, 96 p.

ZUCHOLD Gerd-H., Catalogue Das Erbe der Antike: griechischer und römischer Mythos in der bildenden Kunst der DDR, Berlin [Est] : Staatliche Museen zu Berlin, Abteilung Museumspädagogik, [1980], $36 \mathrm{p}$.

\section{NOTES}

1. «Die Flucht des Sisyphos» [La Fuite de Sisyphe], 1972, huile sur panneau de fibres dur, 96 x $118 \mathrm{~cm}$, Dresde : Galerie Neue Meister.

«Sisyphos behaut den Stein» [Sisyphe taille le rocher], 1974, huile sur panneau de fibres dur, 96 x 119,5 cm, Dresde : Galerie Neue Meister. 
«Der übermütige Sisyphos und die Seinen» [Sisyphe impétueux et les siens], 1976, huile sur toile de lin, 200 x $200 \mathrm{~cm}$, Dresde : Galerie Neue Meister.

2. C'est à partir du début des années 70 que des peintres comme Wolfgang Mattheuer ouvrent des classes de peinture à l'Académie des Arts visuels de Leipzig (Hochschule für Graphik und Buchkunst), où prédominait jusqu'alors le genre de la gravure et de l'illustration de livres. En 1972, le critique d'art est-allemand Lothar Lang introduit l'expression "École de Leipzig", longtemps rejetée par les artistes concernés. Celle-ci renvoie à l'ensemble de la scène artistique de Leipzig depuis le début des années 60 , sans référence à un style unique, ou encore à une forme d'homogénéité - l'École de Leipzig ne comporte pas de phases de création artistique commune. La notion, une création est-allemande, renvoie donc plutôt à une appartenance géographique et non stylistique.

3. Les communications prononcées lors de ce congrès ont été publiées dans la revue de l'Université de Iéna en 1969 [Wissenschaftliche Zeitschrift der Friedrich-Schiller-Universität Jena vol. 18, 1969, n4].

4. Précisions communiquées par Dr. Stephanie-Gerrit Bruer, curateure de la société Winckelmann (échange du 04.03.2009).

5. Arlt Peter, «Der verwitterte Herkules und die Suche in der Trojaburg: Überlegungen zur Bildtypologie und zum Denken in Bildern", in Antikerezeption heute: Protokoll eines Kolloquiums, Stendal : Winckelmann-Museum, 1985, p. 101-108, (Beiträge der Wickelmann-Gesellschaft ; 13).

6. Kunze Max, «Ikarus: zu Aspekten der Antikrezeption in der bildenden Kunst der DDR», in ibid., p. 109-116.

7. Stolzenhain Tilo, «Metamorphosen: Bilder zu Ovis: Gedanken über Kunstwerke zu literarischen Vorlagen», in ibid., p. 115-119.

8. Kuhirt Ullrich, «Ein ungewöhnlicher Realismus?», in Bildende Kunst, n6, 1975, p. 281-285.

9. Catalogue Weggefährten - Zeitgenossen, Berlin-Est : Staatliche Museen, 1979, p. 122.

10. Zuchold quitte la RDA en 1982.

11. Zuchold Gerd H., "Zur Einführung”, in Catalogue Das Erbe der Antike: griechischer und römischer Mythos in der bildenden Kunst der DDR, Berlin [Est]: Staatliche Museen zu Berlin, Abteilung Museumspädagogik, [1980], p. 2-9.

12. Durant la deuxième moitié des années 70 , cette tendance se stabilise. Des artistes de l'avantgarde, comme Hermann Glöckner, désigné par le critique d'art français Raoul-Jean Moulin comme " patriarche de la modernité », seront enfin reconnus officiellement et intégré(e)s dans l'histoire de l'art est-allemande.

13. Voir aussi : Wolle Stefan, Die heile Welt der Diktatur, Bonn : bpb, 1999, p. 285.

14. Le rocher peut être interprété, au sens lacanien, comme symbole du désir.

15. Voir aussi : Collard Maggy, «Sisyphe : Histoire d'une liberté », in Folia Electronica Classica, $\mathrm{n}^{\circ} 3$, jan-juin 2002. [http://bcs.fltr.ucl.ac.be/fe/03/Sisyphe.html]

16. Personare [lat] = parler à travers [fre].

17. En 1974, la RDA organise la $26^{\mathrm{e}}$ assemblée générale de l'AICA [Association Internationale des Critiques d'Art. Consultation des documents : Archives de la Critique d'Art à Châteaugiron (35)]. À cette occasion, Mattheuer sera officiellement présenté à la critique d'art internationale comme un des artistes phares de la RDA.

18. Le nombre de suicides en RDA était globalement très élevé et se concentrait géographiquement dans la région de la Saxe dont Mattheuer est originaire. À partir de 1963, on cessa de publier les statistiques. Durant une période, la recherche médicale sur le suicide fut autorisée en RDA, mais à partir de 1977, les chercheurs se virent refuser l'accès aux statistiques. L'extension de la recherche médicale à ce sujet, au début des années 70, correspond à l'intégration de celui-ci dans la littérature et les arts visuels. On retrouve le thème du suicide en RDA dans le film La Vie des Autres (2006). 
19. Plus de 6000 suicides, soit environ 35 pour 100000 habitants. Voir aussi : Grashoff Udo; Christian Goeschel, «Der Umgang mit Selbstmorden in den beiden Diktaturen in Deutschland», in Diktaturen in Deutschland - Vergleichsaspekte, Bonn : bpb, 2003, p. 476-503. Les œuvres écrites ont pu être censurées par la suppression de quelques passages.

20. La Documenta qui se tient depuis 1955 tous les cinq ans durant cent jours à Kassel en RFA, est une des manifestations mondiales des plus importantes de l'art contemporain. En 1977, 343410 visiteurs s'y sont rendus.

21. Voir aussi : Zuchold Gerd-H., Catalogue Das Erbe der Antike: griechischer und römischer Mythos in der bildenden Kunst der DDR, Berlin [Est]: Staatliche Museen zu Berlin, Abteilung Museumspädagogik, [1980], p. 7.

22. Les grandes exposition sur l'art en RDA se tenaient tous les cinq ans à Dresde $: 7^{\mathrm{e}}$ édition : 1972/73; $8^{\mathrm{e}}$ édition : 1977/78; $9^{\mathrm{e}}$ édition : 1982/83.

23. «Überraschende Begegnung» [Rencontre déconcertante], 1976, gravure sur bois, 50 tirages, $69,9 \times 48,8 \mathrm{~cm}$

24. Voir aussi : Entretien de l'auteure avec l'artiste réalisé en été 2003.

\section{RÉSUMÉS}

Wolfgang Mattheuer (1927-2004) était un des artistes plasticiens les plus connus de la RDA. Ses œuvres séduisent par leur grand format, la simplicité de moyens techniques et leur contenu subversif. Malgré la popularité de ce peintre de la dite l'École de Leipzig en RDA et à l'étranger, quelques œuvres restent méconnues. Jusqu'à présent, la thématique de Sisyphe dans l'œuvre de Mattheuer n'a pas été traitée de manière approfondie. Sans doute à cause de sa dimension mythologique qui impose une lecture élargie, dépassant le cadre de l'histoire de l'art. Mais certainement aussi, à cause de sa dimension critique et subversive concernant la société de la RDA. Cet article se concentre donc sur trois tableaux de l'artiste, jusqu'alors peu commentés par des historien(ne)s ou critiques d'art et qui sont uniques dans le paysage artistique de la RDA par le choix de la thématique antique : Sisyphe.

Wolfgang Mattheuer (1927-2004), member of the so-called School of Leipzig, was one of the most famous artists of the GDR. His works are fascinating thanks to their large format, the simplicity of his artistic techniques and their subversive content. Despite his popularity in the GDR and abroad, some of his works remain relatively unknown. Up until now, the investigation of the myth of Sisyphus in Mattheuer's work has remained relatively superficial, partly because the traditional approaches in art history are too narrow and partly because, in the 1970s, an interdisciplinary interpretation would have revealed critical and subversive content undesirable in the GDR.This article will discuss three paintings by the artist which have until now been rarely studied by art historians and critics. These paintings are unique in the artistic landscape of the 1970s in the GDR due to their choice of a theme from antiquity: the myth of Sisyphus.

«Wolfgang Mattheuer (1927-2004) galt als einer der bekanntesten Künstler der DDR. Seine Werke faszinieren durch große Formate, Einfachheit der angewandten künstlerischen Techniken und durch subversive Inhalte. Trotz seines Bekanntheitsgrades innerhalb der DDR sowie auch im Ausland, blieben einige Werke dieses Malers der sogenannten Leipziger Schule dennoch im Hintergrund. Bisher erschien u.a. die Untersuchung des Sisyphosmythos im Werk Mattheuers 
eher oberflächlich, da traditionelle kunsthistorische Herangehensweisen hier nur bedingt anwendbar sind. Eine fachübergreifende Interpretation hätte aber in den 70er Jahren den kritischen und subversiven Inhalt seiner Werke offenbart und das war in der DDR nicht erwünscht. Dieser Artikel analysiert drei Gemälde des Künstlers zum Sisyphosmythos, welche bisher nur unzureichend interpretiert wurden. Außerdem ist die bildkünstlerische Verarbeitung dieses antiken Themas einzigartig für die künstlerische Landschaft der 1970er Jahre in der DDR.»

INDEX

Mots-clés : RDA, peinture

oeuvrecitee Sisyphe

\section{AUTEURS}

\section{STEFANIE SCHWABE}

Université Rennes et CIERA/Paris 